\title{
An observational project for a large class - determination of the duration of the sidereal day
}

\author{
William Tobin ${ }^{1, *},+$ \\ ${ }^{1}$ Retired from: School of Physical and Chemical Sciences, University of Canterbury, Private Bag 4800, \\ Christchurch 8140, New Zealand \\ Present address: 6 rue Saint Louis, 56000 Vannes, France \\ *william@tobin.fr \\ tORCID: 0000-0002-0533-411X
}

\begin{abstract}
Having students confront the real night sky is difficult for a large class. The determination of the duration of the sidereal day is, however, a project that students can do on their own without specialized equipment. This project provides an introduction to how observational science is done because students must devise the observational procedure, make timings, analyse them, and present results including uncertainties in a report. The experience of running this project as part of a first-year university introduction to the grand ideas of physics is described, with suggestions for improvements and to reduce cheating. Almost a third of students reported results within 1 second of the accepted value.
\end{abstract}

Keywords: Sidereal day; Observational project; Large classes

I saw Eternity the other night,

Like a great ring of pure and endless light,

All calm, as it was bright;

And round beneath it, Time in hours, days, years,

Driv'n by the spheres

Like a vast shadow mov'd; in which the world

And all her train were hurl'd.

- From The World, by the Welsh metaphysical poet Henry Vaughan (1621-1695), used as an epigraph in a student report

\section{Introduction}

Observational work involving quantitative results presents problems for the teacher of introductory astronomy. Having students themselves make observations which they will subsequently analyse usually requires expensive and delicate equip- ment, long bouts of supervision outside normal teaching hours, and contingency arrangements in case of cloud. These difficulties are multiplied if the class is large. Yet an introductory astronomy class in which the student does not confront the real sky is hollow.

There are, however, some projects that students can undertake on their own with minimal equipment. Observations of the lengths of midday shadows is one (Jackson, 2004; Kwok, 2004) but requires student presence around noon, which can lead to timetable clashes in a higher-education context. Understanding variations of the lunar diameter, measured using a homemade sighting device (Krisciunas, 2010), is probably too complex - and the duration of observations too long - for an introductory class. In this note, I describe a short and conceptuallysimple project to determine the duration of the sidereal day that I ran for six years (1988-1993) as part of the term-long 'observable universe' component of a 'grand ideas of physics' first-year course for arts and science students at the University of Canter- 


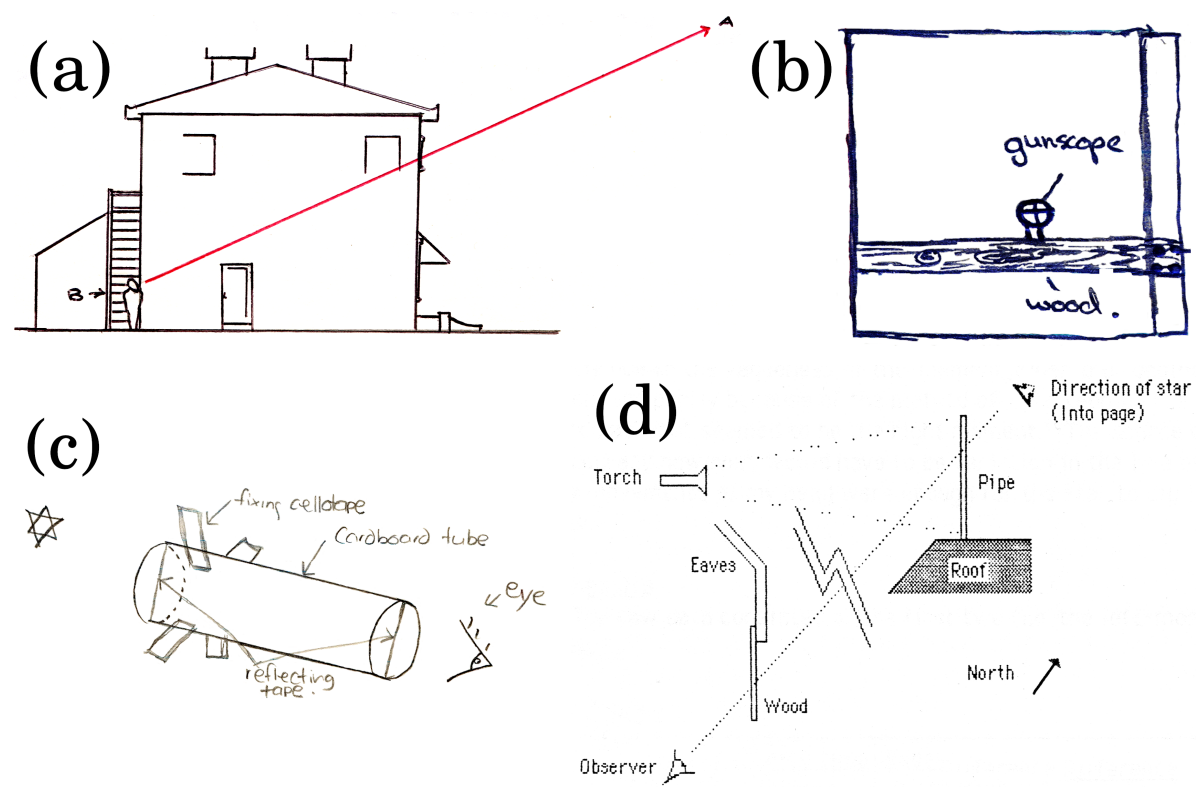

Figure 1. Various student sight lines. (a) The north-south side of a house. (b) A telescopic rifle sight lashed to a wooden bar nailed across a windown frame. Considerable relashing was necessary to centre the target star (Rigel) in the gun sight. (c) The parallel edges of reflecting tape across the ends of a toilet roll, with the roll itself taped to a window. Though this had the advantage of an $\sim 20^{\circ}$ altitude range for chosing the target star, the short tube length produced inaccurate results (d) Alignment between a wooden batten nailed to the eaves of the student's house and an overflow pipe many metres away on a neighbour's roof. The pipe needed illumination with a torch to be visible. Now that cameras are easily available in mobile phones, student reports will doubtless often include photographs of the sight line.

bury in Christchurch, New Zealand. The full course involved two lectures per week for three terms, or about 54 lectures in total, and no backround in science or mathematics was assumed beyond early secondary school. Class size was about one hundred, and from the instructor's point of view, the only effect of a larger or smaller number would be on the time required for marking.

For northern-hemisphere observers Monson (1992) suggests finding the sidereal-day duration by plotting the positions of Polaris and circumpolar stars over two or more hours using a plastic sheet taped against a north-facing window. 'Careful students can easily come within 15 minutes of the accepted value of $23^{\mathrm{h}} 56^{\mathrm{m}}$, ' he states. For those with a reflex camera, star trails provide an analogous method (Royal Observatory Greenwich, 2015). Eckroth (1996) describes using a fixed telescope and an equatorial star to determine a value close to the expected duration.

The approach outlined here is similar to Eckroth's, but requires no telescope. It aims to give students a taste of how quantitative science is done. Besides forcing the students to confront the sky, the project provides an introduction to the process of experimental science. The student must use initiative and pay attention to detail in planning, learn to recognize a brightish star, and make half-a-dozen or so timings. The project requires simple graphical analysis and the presentation of results and uncertainties in a report. I shall suggest how some of the problems encountered three decades ago can now be mitigated thanks to technological advances.

\section{Instructions to Students}

The sidereal-day duration is determined by timing the transit of a given star across a fixed sight line over several nights. Part of a lecture and a three-page handout provided guidance to students.

\subsection{Star and sight line}

Each student's first task was to identify a recognisable star and find or set up a suitable sight line that it will cross when he or she expects to be free from other obligations such as residencehall dinner (or the pub). For greatest accuracy, the sight line should be on the meridian and the star on the celestial equator. This equatorial requirement was not suggested so as not to exclude sight lines to the south for which the stars of Crux and the pointers might be known to the students. Nevertheless, some students realized the desirability of an equatorial star. It was suggested that about half a dozen timings should be taken over an interval of about three weeks. In Aotearoa/New Zealand (the Land of the Long White Cloud), poor weather (and social commitments!) can easily extend this interval, against which eventuality students were asked to ensure that their sight line would still be crossed during the hours of darkness. Students were asked to identify their star on a star chart, not least to avoid any confusion with planets, which would yield incorrect results.

Students showed considerable ingenuity in finding their sight lines (Fig. 1). The corners of university buildings and a reproducible observation point provided many. North-south building walls furnished others. Chimney stacks, water towers and lamp posts were employed. In New Zealand many students live at home, and they enjoyed wider possibilities, such as stakes attached to fences or driven in the ground, or nails hammered into walls, or sighting through plastic pipes. In a land where hunting is common, several students were able to use telescopic rifle sights. The individual nature of each student's star and sight line was of course a brake impeding copying of other students' observations.

As a whole, students were not good at identifying their target stars by name. Choosing a star and sight line are not independent tasks and many target stars were doubtless not the brightest ones that appear on simple star charts. Stars that students did identify by name were bright ones: Regulus, Betelgeuse, Rigel, Alphard, Sirius, Canopus and $\alpha \& \beta$ Crucis, though students noted that with a very bright star, like Sirius, its glare could make timing difficult. Nowadays, the identification prob- 
lem is solved, as almost all students own orientation-sensitive smartphones. Star identification is easy with freeware versions of applications such as SkyView, which shows and identifies the stars towards which the 'phone is pointing, down to fainter than 4th magnitude.

Sight line and star selected, students could encounter unexpected difficulties, such as chalk marks that washed off in the rain, or rugby-club floodlights that rendered the target star invisible.

\subsection{Timing}

Students were asked to ensure that their timepiece was always set to accurate time. For students in the late 1980s/early 1990s that meant repeated comparisons with radio or TV time signals, or the then-new talking-clock service or teletext clock. Nowadays, accurate time is more easily available. Smartphones synchronize to standard time by a variety of means: from the mobile network, from GPS satellites or if connected to the internet via the Network Time Protocol (NTP), as do web sites such as time.is, clock.zone and time.gov. Students were asked to state in their reports how they ensured timings linked to standard time. Some missed the important point that their timepiece needed to be synchronized repeatedly because a watch that gains or loses a few seconds a day leads to a result incorrect by this amount. This is unlikely to be a problem now.

When making their timings, students needed to state their criterion for establishing the instant of crossing and the uncertainty they attached to it. Was one eye or two used? Averted vision? One student's sight plane was defined by a window frame and a distant lamp post, and refined by a needle with a large eye that was stuck into the window frame. The student's eye was positioned so that timings were made when the star filled the needle's eye. Depending on how careful they were, students estimated the uncertainty in a single timing to be from 5 to 30 seconds. Those using a telescope or gun sight estimated 1 or 2 seconds.

\subsection{Analysis}

The students were asked to plot their crossing times against date. The (negative) slope, in seconds per day, indicates how much shorter the sidereal day is than 24 hours. Students were

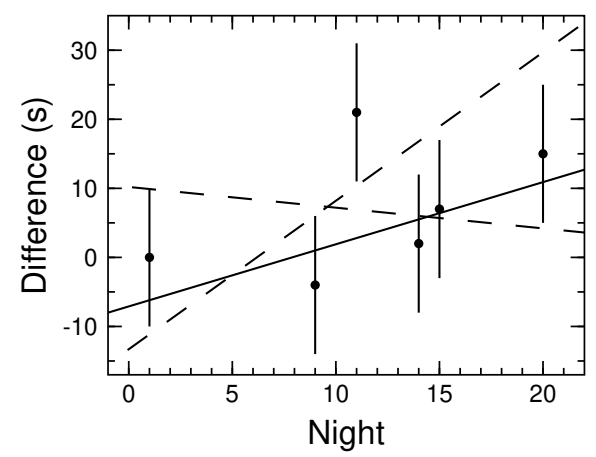

Figure 2. This student's crossing times plotted against night number yielded a whole-second sidereal-day duration of $23^{\mathrm{h}} 56^{\mathrm{m}} 03^{\mathrm{s}}$. This second plot compares the crossing times against those expected using this first-estimate duration. The full line is the student's by-eye estimate of the best fit and has a slope of 0.9 s/day. The student (the same as in Fig. 1 (d)) estimated timings uncertain by $10 \mathrm{~s}$, which from their scatter appears to be a slight over-estimate, and excluded the timing on night 11 which he felt indicated some blunder. The dashed lines show the extreme slopes the student considered just consistent with the timings, yielding a final estimate for the duration of the sidereal day of $23^{\mathrm{h}} 56^{\mathrm{m}} 03.9^{\mathrm{s}+1.3}$
Table 1. Marking scheme.

\begin{tabular}{lc}
\hline Item & $\%$ \\
\hline Introduction and explanation of the sidereal day & 10 \\
Description of the observational procedure, including & 25 \\
$\quad$ orientation of the sight line, choice of star and timing & \\
Presentation of data & 10 \\
Analysis of observations & 15 \\
Discussion of individual and final uncertainties & 15 \\
Suggested improvements & 15 \\
Clarity of exposition and analysis & 5 \\
English & 5 \\
\hline
\end{tabular}

not expected to know about linear regression (although the occasional one did), so unless a student had been very careless, this first, hand-plotted graph did not provide enough resolution to extract the full accuracy of the measurements: a pencil line would be thicker than the uncertainties in the timings. Students were therefore asked to round their derived slope to a whole number of seconds and to calculate for each observation (i) the crossing time predicted assuming this rounded slope, and (ii) by how much the observed crossing time differed from this prediction. Plotted against date, the slope of this second graph of differences provided a correction. In addition, this second graph allowed the exclusion of clearly discrepant observations. The timing uncertainty of the retained observations and their scatter were used to provided an estimate of the uncertainty in the final result via lines with a greater and a lesser slope that the students felt were still, just, compatible with their observations (Fig. 2).

Many students found this analysis difficult, and there were some suprising pitfalls. A few students were confused by the change from daylight-saving to standard time in those years when it occured during the project. Some less-numerate students thought that the interval between day 1 and day $N$ was $N$, not $N-1$. Another common error came in the calculation of expected crossing times. Students usually chose one of their actual timings to correspond to zero difference, but did not appreciate that this was a point just like any other in the second graph. Students did not always realize that expected times needed to be calculated from the round-number slope rather than the accepted value of the sidereal day - though in practice, of course, this is a self-correcting error. Further comments follow in Section 3.1 .

Students were asked to write a report presenting their work, including suggestions for improvements. It was suggested this report did not need to be as long as 2000 words,

\section{Student results}

\subsection{The duration of the sidereal day}

Grading the first student reports in 1988 I immediately encountered two difficulties. First, it was necessary to devise a marking scheme to provide grading uniformity (Table 1). Secondly, checking the analysis in a hundred or so reports was impossibly time consuming. It was necessary to write an interactive, linear-regression computer program into which I could quickly input each student's observations in order to see if the claimed results were in accord with the reported timings. The program made it easy to exclude any outlier points.

Fig. 3 (a) shows the results and uncertainties claimed by students in the 1991 project year. As in earlier years, the majority of students produced results close to the accepted duration of $23^{\mathrm{h}} 56^{\mathrm{m}} 04.0905^{\mathrm{s}}$.

A handful of results clustered near $23^{\mathrm{h}} 55^{\mathrm{m}} 50^{\mathrm{s}}$. The cause 

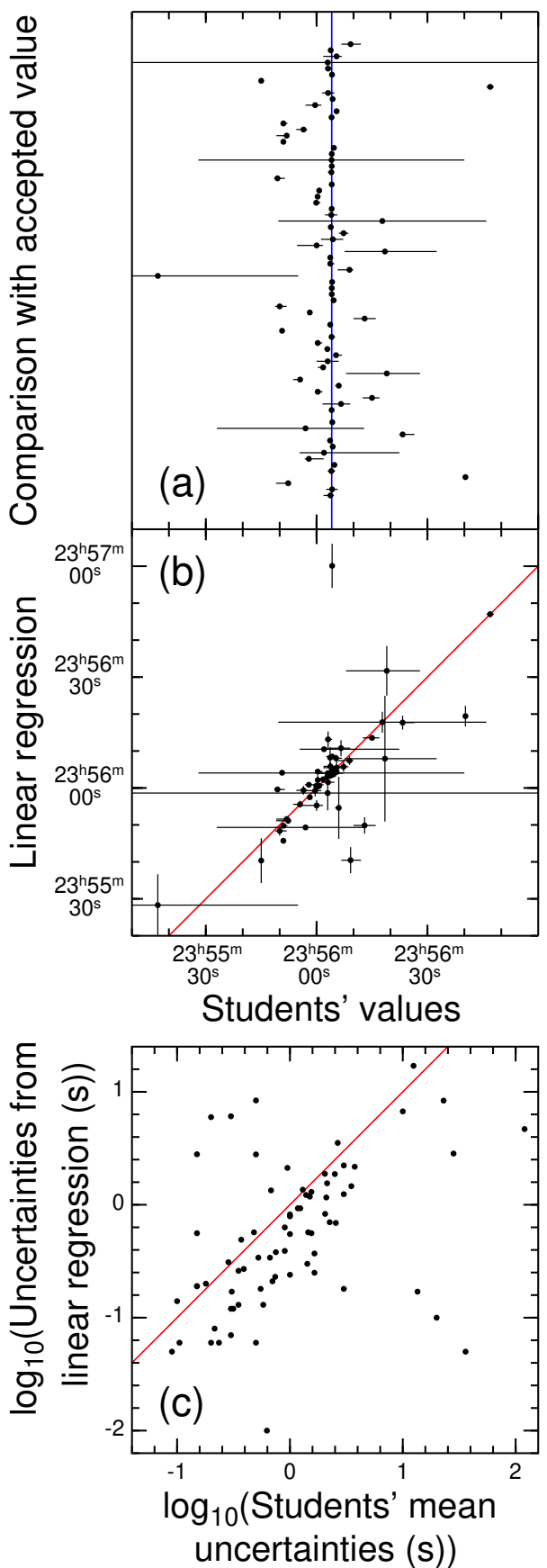

Figure 3. Results from 1991. Not plotted are one result far outside the box and four where no uncertainty was estimated. (a) Stack plot of the sidereal-day length and its uncertainty, as derived by the students. The claimed uncertainty is often smaller than the symbol size. The blue vertical line indicates the ac cepted value of $23^{\mathrm{h}} 56^{\mathrm{m}} 04.1^{\mathrm{s}}$. Forty-seven out of 74 results fall within $\pm 4 \mathrm{~s}$ of this value; 39 within $2 \mathrm{~s}$; and 32 within $1 \mathrm{~s}$. Students using gun sights produced results accurate to $0.1 \mathrm{~s}$. There is a small cluster of results around $23^{\mathrm{h}}$ $55^{\mathrm{m}} 50^{\mathrm{s}}$, discussed in Section 3.1. (b) The students' reported sidereal-day duration compared with my linear-regression analysis of their timings. The red diagonal line indicates equality. The values differ by less than $\pm 4 \mathrm{~s}$ in 53 out of 74 cases. (c) It is difficult to compare the students' final uncertainties with those I derived from linear regression in panel (b), so here they are plotted on logarithmic scales, where the red diagonal line indicates equality.

was as follows. To illustrate the graphical analysis, invented timings were presented and analysed in the handout. They produced a sidereal-day duration close to $23^{\mathrm{h}} 55^{\mathrm{m}} 50^{\mathrm{s}}$. Despite the fact that it was stated in bold face that these timings and the result were entirely fictitious, some students evidently thought their own result should accord with the example.

Did some students delude themselves, mistakenly expecting crossing times $\sim 4^{\mathrm{m}} 10^{\mathrm{s}}$ earlier each night? No doubt some did, but over three weeks that corresponds to an accumulated error of an improbable 5 minutes. A few students clearly got lost in the graphical analysis and sexagesimal arithmetic, with derived durations far from those implied by their reported timings (Fig. 3 (b)). However, a rump of students evidently falsified their timings, reporting ones that were highly consistent with the fictitious $23^{\mathrm{h}} 55^{\mathrm{m}} 50^{\mathrm{s}}$ value given in the handout. Another fraud was clear when a student looked up the expected solarsidereal difference correctly, but applied it in the wrong sense in his fabricated observations, producing a target star that crossed later each night. Perhaps falsified timings are unsurprising. Inventing and altering data were among the top cheating activities amongst English university students reported by Newstead et al. (1996).

To counter all this, I strengthened the handout wording and lecture presentation, suppressing words such as fictitious, which it transpired were unknown to some students. I also asked students to present their project report in two halves, the first with their timings, so they would be committed to them, and the second with their analysis. The number of results near $23^{\mathrm{h}} 55^{\mathrm{m}} 50^{\mathrm{s}}$ declined from its peak in the first year but was not completely extinguished, as Fig. 3 (a) testifies. One must also wonder how many students were savvy enough to escape detection with fabricated timings based on the true sidereal-day duration.

Nowadays, with easy internet connectivity, it would be possible for the instructor to set up an interactive web page on which students would report their crossing times within, say, 48 hours of making them. This should reduce the prevalence of fraud. In addition, various checks could be programmed. Are observations reported for nights that were cloudy? If the target star is known, does the the crossing time correspond to the azimuth of the claimed sight line? If by-hand analysis is felt to be too much for the students, the analysis could be programmed into the web page, or effected with other tools such as spreadsheet software like Excel.

Figure 3 (c) compares the uncertainties stated by the students with those I obtained by linear regression. There are many cases where the students have significantly under- or over-estimated their uncertanties, but it is striking that most are essentially in agreement with the linear-regression values, although larger by a factor of about two ( 0.3 in the logarithm). The students were asked to estimate the range of sidereal-day durations just consistent with their data. The mean uncertainty plotted here is half this range and obviously this 'two- $\sigma$ ' value would be expected to be about twice that obtained by linear regression, as seen.

One year a number of students stated that the accepted length of the sidereal day was $23^{\mathrm{h}} 56^{\mathrm{m}} \mathrm{O} 3^{\mathrm{s}}$, which on investigation was the erroneous value given by one of the astronomy text books in the University Library (Jastrow and Thompson, 1984). An occasional, well-informed student noted that because of the precession of the equinoxes the sidereal day is shorter than the rotation period of the Earth by $0.0084 \mathrm{~s}$.

\subsection{Suggested improvements}

Students suggested a number of possible improvements, such as a head brace to keep the eye still, working in pairs with one student watching the star and the other the time, working in the less-cloudy summer months, working away from the city and its lights, using a watch with a lighted dial, using a fixed telescope or gun sight, observing over a longer interval - and restricting social life! One student invoked the possibility of photographing star trails. A history student with math anxiety sug- 
gested help from a more-numerate friend. 'Finally, if I were to repeat this experiment,' wrote another student, 'I would not take my readings outside a house that has a great big dog stationed on the front lawn.'

\section{Conclusion}

Asking students to determine the duration of the sidereal day is a project that is suited to large classes, and could even be used in on-line courses. Besides introducing students to the sky, it parallels real research - devising an observational procedure, making observations, analysing them, and reporting the results. These are of course useful skills beyond introductory astronomy. As with all independent work, there are opportunities for cheating but they can be reduced by using the strategies outlined.

Many students were thrilled by their interaction with the real night sky. 'This project was, in my opinion, very exciting and satisfying,' wrote one. As a university teacher, I have always thought, to paraphrase Plutarch, that education is the kindling of a flame, not the filling of a vessel (Babbitt, 1927). I was particularly touched by the words of another student: 'This project has made me stop and take stock,' she wrote. 'Previously I thought that a day was a day, 24 hours, now I have found another type. I wonder how many more things I take for granted have hidden or partly hidden companions!!'

\section{References}

Babbitt, F. C. (1927). Plutarch's Moralia, volume I. Harvard University Press, 1st edition. 'On Listening to Lectures,' at p. 257.

Eckroth, C. A. (1996). Observations of the sidereal day. The Physics Teacher, 34:94. https://doi.org/10.1119/1.2344355.

Jackson, E. (2004). Daytime astronomy in the northern hemisphere using shadows. Astronomy Education Review, 2:146-149. https://doi.org/10.3847/AER2003021.

Jastrow, R. and Thompson, M. H. (1984). Astronomy: Fundamentals and Frontiers. Wiley, 4th edition. p. I-26.

Krisciunas, K. (2010). Determining the eccentricity of the Moon's orbit without a telescope. American Journal of Physics, 78:834-838. https://doi.org/10.1119/1.3369968.

Kwok, P.-W. (2004). Studying the transit of the Sun using shadows. Astronomy Education Review, 3:106-114. https://doi. org/10.3847/AER2004010.

Monson, B. (1992). A simple method of measuring the length of the sidereal day. The Physics Teacher, 30:558-559. https: //doi.org/10.1119/1.2343640.

Newstead, S. E., Franklyn-Stokes, A., and Armstead, P. (1996). Individual differences in student cheating. Journal of Educational Psychology, 88:229-241. https://doi.org/10.1037/ 0022-0663.88.2.229.

Royal Observatory Greenwich (2015). Using star trails to measure the sidereal day. Retrieved from https: //www.rmg.co.uk/sites/default/files/KS4\%20Star\%20Trails\% 20and\%20Sidereal\%20Day. pdf (accessed 2021 January 14). 